\title{
Newton Interpolating Series at $m$ Distinct Points with Coefficients in a Real Banach Algebra
}

\author{
Ghiocel Groza $^{1}$ and Nicolae Pop ${ }^{2}$ \\ ${ }^{1}$ Department of Mathematics and Informatics, Technical University of Civil Engineering, \\ 124 Lacul Tei, 020396 Bucharest, Romania \\ ${ }^{2}$ Department of Mathematics and Computer Science, North University of Baia Mare, Victoriei 76, \\ 430122 Baia Mare, Romania \\ Correspondence should be addressed to Ghiocel Groza, grozag@utcb.ro
}

Received 28 December 2010; Accepted 18 February 2011

Academic Editor: Heinrich Begehr

Copyright (C) 2011 G. Groza and N. Pop. This is an open access article distributed under the Creative Commons Attribution License, which permits unrestricted use, distribution, and reproduction in any medium, provided the original work is properly cited.

A real Banach algebra of Newton interpolating series, used to approximate the solutions of multipoint boundary value problems for ODE's, is studied.

\section{Introduction}

Let $(\mathcal{A},\|\|)$ be a real Banach algebra. In this paper we study the so-called Newton interpolating series having the coefficients in $(\mathcal{A},\|\|)$, defined in Section 2 , by means of Newton interpolating polynomials at $m$ distinct nodes, $x_{0}, x_{1}, \ldots, x_{m-1}$, where $m \geq 2$, of multiplicity $n_{i}, i=0,1, \ldots, m-1$. These series are useful generalizations of power series which, in particular forms, were used in number theory to prove the transcendence of some values of exponential series [1, Chapter 2]. Knowing the importance of power series in the theory of initial value problems for differential equations, it seems to be useful to study Newton interpolating series in order to find the solution of the multipoint boundary value problems for differential equations. In [2] there are some numerical results in the particular case $\mathcal{A}=\mathbb{R}$. Other methods used to solve similar interpolation problems can be found in [3].

In Section 2 we obtain results concerning Newton interpolating series and their derivatives (Theorems 2.1 and 2.2). Section 3 deals with functions which are representable into Newton interpolating series. Theorem 3.4 gives a criterion for a function to be representable into a Newton interpolating series (see Remark 3.7). The coefficients of this series are computed in Theorem 3.1. We prove that, with respect to a suitable norm, the algebra of these functions becomes a real Banach algebra (Theorem 3.6). This adds a new 
completion of the ring of polynomials $\mathcal{A}[x]$, where $x$ commutes with the elements of $\mathcal{A}$, to the usual real Banach spaces, where $\mathcal{A}[x]$ is a dense subset. Other examples can be found in [4].

\section{Newton Interpolating Series at $m$ Distinct Points}

Let $x_{0}, x_{1}, \ldots, x_{m-1}$ be a set of real numbers which satisfy $x_{0}<x_{1}<\cdots<x_{m-1}$, where $m \geq 2$. If $i$ is a nonnegative integer, then we consider the quotient $q(i)$ and the remainder $r(i)$ obtained when $i$ is divided by $m$. We construct the polynomials

$$
u_{i}(x)=\prod_{k=0}^{m-1}\left(x-x_{k}\right)^{q(i)} \prod_{k=0}^{r(i)-1}\left(x-x_{k}\right), \quad i=0,1,2, \ldots
$$

where $x$ is a real variable. Hence

$$
u_{i}(x)=u_{m}^{q(i)}(x) u_{r(i)}(x)
$$

If $(\mathcal{A},\|\|)$ is a real Banach algebra, we call an infinite series of the form

$$
\sum_{i=0}^{\infty} A_{i} u_{i}(x)
$$

where $A_{i} \in \mathcal{A}$ and $x$ commutes with the elements of $\mathcal{A}$, a Newton interpolating series with coefficients in $\mathcal{A}$ at $x_{0}, x_{1}, \ldots, x_{m-1}$. We choose a real number $x$ and consider the partial sum $S_{n, x}=\sum_{i=0}^{n} A_{i} u_{i}(x)$. The series (2.3) is called convergent at $x$ if there exists $S_{x} \in \mathcal{A}$ such that the sequence $\left\{S_{n, x}\right\}_{n \in \mathbb{N}}$ converges to $S_{x}$ in $\mathcal{A}$ that is $\lim _{n \rightarrow \infty}\left\|S_{x}-S_{n, x}\right\|=0$. If $I$ is a set and the series is convergent at $x$, for every $x \in I$, then we say that the series converges on $I$. In this case the function $S: I \rightarrow \mathcal{A}$, given by $S(x)=S_{x}$, is called the sum of the series (2.3) on $I$.

Theorem 2.1. Let $(\mathcal{A},\|\|)$ be a real Banach algebra. If a Newton interpolating series of the form (2.3) converges at a real number $\bar{x}$ and $\left|u_{m}(\bar{x})\right| \neq 0$, then there exists an interval $I_{\bar{x}}$ which contains $\bar{x}$ and at least one of $x_{i}$ such that the series converges absolutely on $I_{\bar{x}} \backslash\{\bar{x}\}$ and converges uniformly on every closed interval $[a, b] \subset I_{\bar{x}}$.

Proof. Since $u_{m}(x)$ is a polynomial of degree $m$ having $m$ distinct real roots, it follows that there exists an interval $I_{\bar{x}}$ which contains $\bar{x}$ and at least one of $x_{i}$ such that

$$
\left|u_{m}(x)\right|<\left|u_{m}(\bar{x})\right|
$$

for every $x \in I_{\bar{x}} \backslash\{\bar{x}\}$. Because the series converges at $\bar{x}$, there exists $M>0$ such that, for every $i$,

$$
\left\|A_{i} u_{i}(\bar{x})\right\|<M
$$


If $x \in I_{\bar{x}}$, then according to (2.2) and (2.5)

$$
\left\|A_{i} u_{i}(x)\right\|<M v^{i-r(i)}(x)\left|\frac{u_{r(i)}(x)}{u_{r(i)}(\bar{x})}\right|
$$

where

$$
v(x)=\left|\frac{u_{m}(x)}{u_{m}(\bar{x})}\right|^{1 / m}
$$

Let

$$
M^{\prime}=\max _{0 \leq r \leq m-1}\left|\frac{u_{r}(x)}{u_{r}(\bar{x})}\right| .
$$

Now, from (2.4), (2.6), and (2.8), we obtain $\left\|A_{i} u_{i}(x)\right\|<M M^{\prime} v^{i-m}(x), i=0,1, \ldots$, where $v(x)<1$. Hence, the series converges absolutely at $x$.

If $[a, b] \subset I_{\bar{x}}$, then we consider $x \in[a, b]$. Let

$$
M^{\prime \prime}=\max _{0 \leq r \leq m-1} \max _{x \in[a, b]}\left|\frac{u_{r}(x)}{u_{r}(\bar{x})}\right| .
$$

Then there exists $c \in[a, b]$ such that, for every $x \in[a, b]$,

$$
v(x) \leq v(c)<1
$$

and $\left\|A_{i} u_{i}(x)\right\| \leq M M^{\prime \prime} v^{i-m}(c)$. Thus, from (2.9), (2.10), and Weierstrass test, the series is uniformly convergent on $[a, b]$.

Given a Newton interpolating series of the form (2.3), we consider the polynomials

$$
\begin{aligned}
& P_{1, m}(x)=1, \\
& P_{i, m}(x)= \begin{cases}u_{i}^{\prime}(x), & \text { if } i \leq m, \\
(q(i)+1) u_{r(i)}^{\prime}(x) v_{r(i)}(x)+q(i) u_{r(i)}(x) v_{r(i)}^{\prime}(x), & \text { if } i>m,\end{cases}
\end{aligned}
$$

where

$$
v_{r(i)}(x)=\prod_{k=r(i)}^{m-1}\left(x-x_{k}\right)
$$


If $t \leq m$, then we denote by $P_{i, m ; t}\left(z_{1} ; z_{2} ; \ldots ; z_{t}\right)$ the divided difference of $P_{i, m}(x)$ with respect to $t$ distinct points $z_{1}, z_{2}, \ldots, z_{t}$. Thus, $P_{i, m ; 1}\left(z_{1}\right)=P_{i, m}\left(z_{1}\right)$ and for $j \geq 2$

$$
P_{i, m ; j}\left(z_{1} ; z_{2} ; \ldots ; z_{j}\right)=\frac{P_{i, m ; j-1}\left(z_{2} ; \ldots ; z_{j}\right)-P_{i, m ; j-1}\left(z_{1} ; \ldots ; z_{j-1}\right)}{z_{j}-z_{1}} .
$$

Since, for $i \geq m+1, u_{i}^{\prime}(x)=u_{i-m}(x) P_{i, m}(x)$, then by (2.1), (2.2), (2.11) and by Newton's interpolation formula, it follows that

$$
u_{i}^{\prime}(x)= \begin{cases}\sum_{k=1}^{i} P_{i, m ; k}\left(x_{0} ; x_{1} ; \ldots ; x_{k-1}\right) u_{k-1}(x), & \text { if } i \leq m, \\ \sum_{k=1}^{m} P_{i, m ; m-k+1}\left(x_{r(i)} ; \ldots ; x_{r(i)+m-k}\right) u_{i-k}(x), & \text { if } i \geq m+1,\end{cases}
$$

where, for $s \geq m, x_{s}=x_{r(s)}$.

The series

$$
\sum_{i=0}^{\infty} A_{i}^{(1)} u_{i}(x)
$$

where, if $i \leq m-1$,

$$
\begin{aligned}
A_{i}^{(1)}= & \sum_{j=1}^{m-i} P_{i+j, m ; i+1}\left(x_{0} ; x_{1} ; \ldots ; x_{i}\right) A_{i+j} \\
& +\sum_{j=m-i+1}^{m} P_{i+j, m ; m-j+1}\left(x_{r(i+j)} ; \ldots ; x_{r(i+j)+m-j}\right) A_{i+j}
\end{aligned}
$$

and, if $i \geq m$,

$$
A_{i}^{(1)}=\sum_{j=1}^{m} P_{i+j, m ; m-j+1}\left(x_{r(i+j)} ; x_{r(i+j)+1} ; \ldots ; x_{r(i+j)+m-j}\right) A_{i+j}
$$

is called the derived series of (2.3).

Now, let us denote by $\mathcal{L}_{n}(\mathbb{R}, \mathcal{A})$ the complete real algebra of bounded multilinear forms $f: \mathbb{R}^{n} \rightarrow \mathcal{A}$. Then we can consider $\mathcal{A}=\mathcal{L}(\mathbb{R}, \mathcal{A})$, and hence $\mathcal{A}=\mathcal{L}_{n}(\mathbb{R}, \mathcal{A})$. Thus, if $I \subset \mathbb{R}$ is an interval and $F: I \rightarrow \mathcal{A}$, then the Fréchet derivatives of this function at a point of $I$ can be considered an element of $\mathcal{A}$.

Theorem 2.2. Let $(\mathcal{A},\|\|)$ be a real Banach algebra. If the series (2.3) converges, on an open interval I which does not contain a local extremum of $u_{m}(x)$, to $S$, then the derived series of (2.3) converges absolutely on $I$, there exists the Fréchet derivative $S^{\prime}(x)$ of $S(x)$ at $x \in I$, and

$$
S^{\prime}(x)=\sum_{i=0}^{\infty} A_{i}^{(1)} u_{i}(x)
$$


where $A_{i}^{(1)}$ are given in (2.16), (2.17). Moreover, the series (2.15) can be obtained by termwise differentiation from the series (2.3).

Proof. If $x \in I$, since $u_{m}(x)$ is a polynomial and $I$ does not contain a local extremum of $u_{m}(x)$, then we can choose $\tilde{x} \in I$ such that

$$
\left|u_{m}(x)\right|<\left|u_{m}(\tilde{x})\right|
$$

Since the series (2.3) is convergent at $\tilde{x}$, there exists $M>0$ such that

$$
\left\|A_{i} u_{i}(\tilde{x})\right\|<M, \quad i=0,1, \ldots
$$

Hence, for $k \in\{0,1, \ldots m-1\}$,

$$
\left\|A_{i+k} u_{i}(x)\right\|=\left\|A_{i+k} u_{i+k}(\tilde{x})\right\|\left|\frac{u_{i}(\tilde{x})}{u_{i+k}(\tilde{x})}\right|\left|\frac{u_{i}(x)}{u_{i}(\tilde{x})}\right| \leq M^{\prime}\left|\frac{u_{i}(x)}{u_{i}(\tilde{x})}\right|
$$

where

$$
M^{\prime}=M \max _{0 \leq n \leq m-1}\left|\frac{u_{i}(\tilde{x})}{u_{i+n}(\tilde{x})}\right|
$$

Here we remark that, from (2.2), for a fixed $\tilde{x}, u_{i}(\tilde{x}) / u_{i+n}(\tilde{x})$ takes only a finite number of values, for $i \in \mathbb{N}$.

Since (see, e.g., [5], page 121)

$$
P_{i, m ; t}\left(z_{0} ; z_{1} ; \ldots ; z_{t-1}\right)=\sum_{k=0}^{t-1} \frac{P_{i, m}\left(z_{k}\right)}{u_{t}^{\prime}\left(z_{k}\right)}, \quad t \leq m,
$$

where we consider $z_{0}<z_{1}<\cdots<z_{m-1}$ and

$$
u_{i}(z)=\prod_{k=0}^{m-1}\left(z-z_{k}\right)^{q(i)} \prod_{k=0}^{r(i)-1}\left(z-z_{k}\right)
$$

by (2.11) and (2.23), for $j=1,2, \ldots, m$, we obtain

$$
P_{i+j, m ; m-j+1}\left(x_{r(i+j)} ; x_{r(i+j)+1} ; \ldots ; x_{r(i+j)+m-j}\right)=\alpha_{i+j} q(i)+\beta_{i+j},
$$

where $\alpha_{i+j}=\alpha_{i+j}\left(x_{0}, x_{1}, \ldots x_{m-1}\right), \beta_{i+j}=\beta_{i+j}\left(x_{0}, x_{1}, \ldots x_{m-1}\right)$ take only a finite number of values. Then there exists $C_{1}>0$ such that, for all $i$ and $j$,

$$
\left|P_{i+j, m ; m-j+1}\left(x_{r(i+j)} ; x_{r(i+j)+1} ; \ldots ; x_{r(i+j)+m-j}\right)\right|<C_{1} q(i) .
$$


By (2.2) there exists $C_{2}=C_{2}(x, \tilde{x})>0$, independent of $i$, such that

$$
\left|\frac{u_{i}(x)}{u_{i}(\tilde{x})}\right|<C_{2}\left|\frac{u_{m}(x)}{u_{m}(\tilde{x})}\right|^{q(i)}
$$

Hence, by using (2.16), (2.17), (2.21), (2.26), and (2.27), it follows that there exists $C_{3}>0$, independent of $i$, such that

$$
\left|A_{i}^{(1)} u_{i}(x)\right| \leq C_{3} q(i)\left|\frac{u_{m}(x)}{u_{m}(\tilde{x})}\right|^{q(i)}
$$

Then, by (2.19), the series (2.15) converges absolutely at $x$ and also on $I$. By (2.14) it follows that, for $x \in I$, the series (2.15) can be obtained by termwise differentiation from the series (2.3).

Now, if $x \in I$, because for $x=x_{i}, S(x)$ is a polynomial function and Theorem 2.2 follows easily, then we may suppose that $u_{m}(x) \neq 0$. Then, by Theorem 2.1, we can choose a compact interval $I_{x} \subset I$ such that $x$ belongs to its interior, $x_{i} \notin I_{x}$, for every $i$, and the series (2.3) converges absolutely on $I_{x}$. We consider $\delta>0$ small enough such that for every $h \in \mathbb{R},|h| \leq \delta, x+h \in I_{x}$. Then

$$
\frac{S(x+h)-S(x)}{h}=\sum_{i=1}^{\infty} \alpha_{i}(h)
$$

where

$$
\alpha_{i}(h)=A_{i} \frac{u_{i}(x+h)-u_{i}(x)}{h}=A_{i} u_{i}^{\prime}\left(\xi_{i}\right)
$$

where $\xi_{i}$ is a point lying between $x$ and $x+h$.

If $y \in I_{x}$, then it is different from $x_{i}$, for every $i$, and, by (2.2),

$$
\begin{aligned}
u_{i}^{\prime}(y) & =q(i) u_{m}^{q(i)-1}(y) u_{m}^{\prime}(y) u_{r(i)}(y)+u_{m}^{q(i)}(y) u_{r(i)}^{\prime}(y) \\
& =\frac{u_{m}^{\prime}(y)}{u_{m}(y)} q(i) u_{i}(y)+\frac{u_{r(i)}^{\prime}(y)}{u_{r(i)}(y)} u_{i}(y) .
\end{aligned}
$$

We put

$$
\begin{array}{cc}
M_{1}=\max _{0 \leq j \leq m} \max _{y \in I_{x}}\left|\frac{u_{j}^{\prime}(y)}{u_{j}(y)}\right|, \quad M_{2}=\max _{0 \leq j \leq m} \max _{y \in I_{x}}\left|u_{j}(y)\right|, \\
m_{1}=\min _{0 \leq j \leq m} \min _{y \in I_{x}}\left|\frac{u_{j}^{\prime}(y)}{u_{j}(y)}\right|, \quad m_{2}=\min _{0 \leq j \leq m} \min _{y \in I_{x}}\left|u_{j}(y)\right| .
\end{array}
$$


Since, for $y \in I_{x}$, the series $\sum_{i=0}^{\infty} A_{i} u_{i}(y)$ converges absolutely, it follows that the series $\sum_{i=0}^{\infty}\left\|A_{i}\right\|\left|u_{i}(y)\right|$ converges. Hence, because $m_{2}>0$, we obtain that the series $\sum_{i=0}^{\infty} A_{i} u_{m}^{q(i)}(y)$ converges absolutely. Similarly, since the series $\sum_{i=0}^{\infty} A_{i} u_{i}^{\prime}(y)$ and $\sum_{i=0}^{\infty} A_{i}\left(u_{r(i)}^{\prime}(y) / u_{r(i)}(y)\right) u_{i}(y)$ converge absolutely, from (2.31), it follows that the series $\sum_{i=0}^{\infty} A_{i}\left(u_{m}^{\prime}(y) / u_{m}(y)\right) q(i) u_{i}(y)$ converges absolutely. Then, by (2.2), the series $\sum_{i=0}^{\infty}\left\|A_{i}\right\| q(i)\left|u_{m}^{q(i)}(y) \| u_{r(i)}(y)\right|$ converges, and thus $\sum_{i=0}^{\infty} A_{i} q(i) u_{m}^{q(i)}(y)$ converges absolutely. We consider $x_{0} \in I_{x}$ such that

$$
\left|u_{m}\left(x_{0}\right)\right|=\max _{y \in I_{x}}\left|u_{m}(y)\right|
$$

and we put

$$
M_{3}=\max _{0 \leq j \leq m} \max _{y \in I_{x}}\left|u_{j}^{\prime}(y)\right|
$$

Then, from (2.31)-(2.35), for every $i$,

$$
\begin{aligned}
\left|u^{\prime}\left(\xi_{i}\right)\right| & \leq q(i) M_{2} M_{3}\left|u_{m}^{q(i)-1}\left(x_{0}\right)\right|+M_{3}\left|u_{m}^{q(i)}\left(x_{0}\right)\right| \\
& \leq \frac{M_{2} M_{3}}{m_{2}} q(i)\left|u_{m}^{q(i)}\left(x_{0}\right)\right|+M_{3}\left|u_{m}^{q(i)}\left(x_{0}\right)\right| .
\end{aligned}
$$

Hence, the series $\sum_{i=0}^{\infty} A_{i} u^{\prime}\left(\xi_{i}\right)=\sum_{i=1}^{\infty} \alpha_{i}(h)$ converges absolutely and uniformly on $[-\delta, \delta]$. If we put $\alpha_{i}(0)=A_{i} u_{i}^{\prime}(x)$, then it follows that the functions $\alpha_{i}(h)$ are continuous on $[-\delta, \delta]$. Thus, the sum of the series (2.29) is continuous and

$$
\lim _{h \rightarrow 0} \frac{S(x+h)-S(x)}{h}=\sum_{i=1}^{\infty} \alpha_{i}(0)=\sum_{i=0}^{\infty} A_{i} u_{i}^{\prime}(x) .
$$

Hence,

$$
S(x+h)-S(x)=\sum_{i=0}^{\infty} A_{i} u_{i}^{\prime}(x) h+o(h)
$$

and this completes the proof of the theorem.

\section{Representable Functions into Newton Interpolating Series at $m$ Distinct Points}

If $I \subset \mathbb{R}$ is an interval which contains $x_{0}, x_{1}, \ldots, x_{m-1}$ such that $x_{0}<x_{1}<\cdots<x_{m-1}, m \geq 2$, then we consider a function $F: I \rightarrow \mathcal{A}$. We say that the function $F$ is representable into $a$ Newton interpolating series at $x_{0}, x_{1}, \ldots, x_{m-1}$ if there exists a series of the form (2.3), which 
converges absolutely on $I$ and converges uniformly to $F$ on $I$. Hence, it follows that $F$ is a continuous function.

When $x_{j}=j+1, I=\mathscr{A}=\mathbb{R}$, and $F(x)=e^{\alpha x}$, it is known that the coefficients $A_{i}$ and $F$ are related through the complex integral formula (see [1], Chapter 2, Section 3)

$$
A_{i}=\frac{1}{2 \pi \sqrt{-1}} \int_{|z|=i} \frac{F(z)}{u_{i+1}(z)} d z
$$

If $F$ is representable into a Newton interpolating series at $x_{0}, x_{1}, \ldots, x_{m-1}$, by using Theorems 2.1 and 2.2, similarly as in [2, Theorem 3], then it follows the following result which gives a formula for $A_{i}$.

Theorem 3.1. Suppose that $F: I \rightarrow \mathcal{A}$ is a function which is representable into a Newton interpolating series $\Sigma$ of the form (2.3) at $x_{0}, x_{1}, \ldots, x_{m-1} \in I$. Then, for every $n$, there exists $J_{n}=\bigcup_{i=0}^{m-1} V_{i, n} \subset I$, where, for every $i, V_{i, n}$ is an open interval which contains $x_{i}, F$ has Fréchet derivative of order $n$ on $V_{i, n}$, the series $\Sigma$ is uniquely defined, and for each $i$

$$
\begin{aligned}
A_{i}= & \frac{1}{q(i) !} \sum_{j=0}^{r(i)}\left(\frac{F(x)}{\prod_{k=0, k \neq j}^{r(i)}\left(x-x_{k}\right) \prod_{k=0, k \neq j}^{m-1}\left(x-x_{k}\right)^{q(i)}}\right)_{x=x_{j}}^{(q(i))} \\
& +\frac{1}{(q(i)-1) !} \sum_{j=r(i)+1}^{m-1}\left(\frac{F(x)}{\prod_{k=0}^{r(i)}\left(x-x_{k}\right) \prod_{k=0, k \neq j}^{m-1}\left(x-x_{k}\right)^{q(i)}}\right)_{x=x_{j}}^{(q(i)-1)},
\end{aligned}
$$

where $(F(x))^{(-1)}$ and $1 /(-1)$ ! mean 0 .

If $F: I \rightarrow \mathcal{A}$ is representable into a Newton interpolating series at $x_{0}, x_{1}, \ldots, x_{m-1} \in I$, then the series of the form (2.3), where $A_{i}$ are given in (3.2), is called the Newton interpolating series at $x_{0}, x_{1}, \ldots, x_{m-1}$ of the function $F$.

Given a Newton interpolating series of the form (2.3), we put

$$
N_{n}(x)=\sum_{i=0}^{n} A_{i} u_{i}(x)
$$

Now, in order to prove a corollary of the theorem, we need the following form of Lagrange's theorem.

Lemma 3.2. One considers a real Banach algebra $\mathbb{A}$ and $\mathcal{A}^{*}$ the dual of $\mathcal{A}$. If $F:[a, b] \rightarrow \mathcal{A}^{*}$, where $a<b$ is Fréchet differentiable on $[a, b]$, then there exists $c \in(a, b)$ such that

$$
F(b)-F(a)=F^{\prime}(c)(b-a) .
$$

Proof. If $x \in[a, b]$ and $h_{1} \in \mathcal{A}$, then we consider the real function $\varphi$ given by $\varphi\left(x, h_{1}\right)=$ $F(x)\left(h_{1}\right)$. Then, for every $h_{1} \in \mathcal{A}, h_{2} \in \mathbb{R}$ such that $x+h_{2} \in[a, b], \varphi\left(x+h_{2}, h_{1}\right)-\varphi\left(x, h_{1}\right)=$ $F\left(x+h_{2}\right)\left(h_{1}\right)-F(x)\left(h_{1}\right)=F^{\prime}(x)\left(h_{2}\right)\left(h_{1}\right)+o\left(h_{2}\right)$ and $\varphi$ has Fréchet derivative with respect to $x$. 
Now, for $h_{1} \in \mathcal{A}$, we consider the real function $g_{h_{1}}:[a, b] \rightarrow \mathbb{R}$, defined by $g_{h_{1}}(x)=\varphi\left(x, h_{1}\right)$. Then, by Lagrange's theorem, there exists $c \in(a, b)$ such that $g_{h_{1}}(b)-g_{h_{1}}(a)=g_{h_{1}}^{\prime}(c)(b-a)$. Hence, the lemma is proved.

Corollary 3.3. Suppose that $F:\left[x_{0}, x_{m-1}\right] \rightarrow A$ has Fréchet derivatives of all orders on $\left[x_{0}, x_{m-1}\right]$ and it is representable into a Newton interpolating series at $x_{0}, x_{1}, \ldots, x_{m-1}$. If its Newton interpolating series (2.3) has the partial sums given in (3.3), then, for a fixed $n$, the polynomial $N_{n}(x)$ satisfies

$$
N_{n}^{\left(\alpha_{j}\right)}\left(x_{j}\right)=F^{\left(\alpha_{j}\right)}\left(x_{j}\right),
$$

where $0 \leq j \leq m-1$ and

$$
\alpha_{j} \in \begin{cases}\{0,1, \ldots, q(n)\}, & \text { if } j \leq r(n), \\ \{0,1, \ldots, q(n)-1\}, & \text { if } j \geq r(n)+1\end{cases}
$$

Furthermore, if $\mathbb{A}$ is the dual algebra of a real Banach algebra $\mathcal{A}_{1}$, then, for every $x \in\left[x_{0}, x_{m-1}\right]$, there exists $\xi=\xi(x, n) \in\left(x_{0}, x_{m-1}\right)$ such that

$$
F(x)=N_{n}(x)+\frac{F^{(n+1)}(\xi)}{(n+1) !} u_{n+1}(x)
$$

Proof. It follows immediately that every polynomial function is representable into a finite Newton interpolating series at $x_{0}, x_{1}, \ldots, x_{m-1}$. Then the coefficients $A_{i}, i \leq n$, are given in (3.2), where $F$ is replaced by $N_{n}$. Since $N_{n}(x)$ are the partial sums of the Newton interpolating series at $x_{0}, x_{1}, \ldots, x_{m-1}$ of the function $F$, it follows that

$$
\begin{aligned}
A_{i}= & \frac{1}{q(i) !} \sum_{j=0}^{r(i)}\left(\frac{F(x)}{\prod_{k=0, k \neq j}^{r(i)}\left(x-x_{k}\right) \prod_{k=0, k \neq j}^{m-1}\left(x-x_{k}\right)^{q(i)}}\right)_{x=x_{j}}^{(q(i))} \\
& +\frac{1}{(q(i)-1) !} \sum_{j=r(i)+1}^{m-1}\left(\frac{F(x)}{\prod_{k=0}^{r(i)}\left(x-x_{k}\right) \prod_{k=0, k \neq j}^{m-1}\left(x-x_{k}\right)^{q(i)}}\right)_{x=x_{j}}^{(q(i)-1)} \\
= & \frac{1}{q(i) !} \sum_{j=0}^{r(i)}\left(\frac{N_{n}(x)}{\prod_{k=0, k \neq j}^{r(i)}\left(x-x_{k}\right) \prod_{k=0, k \neq j}^{m-1}\left(x-x_{k}\right)^{q(i)}}\right)_{x=x_{j}}^{(q(i))} \\
& +\frac{1}{(q(i)-1) !} \sum_{j=r(i)+1}^{m-1}\left(\frac{N_{n}(x)}{\prod_{k=0}^{r(i)}\left(x-x_{k}\right) \prod_{k=0, k \neq j}^{m-1}\left(x-x_{k}\right)^{q(i)}}\right)_{x=x_{j}}^{(q(i)-1)} .
\end{aligned}
$$

Then (3.5) follows, by mathematical induction on $n$, by using (3.8), with $i=0,1, \ldots$ We observe, finally, that (3.7) gives the error of the interpolating formula and it is obtained by 
applying the generalization of Rolle's theorem, which is a consequence of Lemma 3.2, for the function $H(x)=F(x)-N_{n}(x)+K u_{n+1}(x)$, where $K$ is a constant from $\mathcal{A}$.

Theorem 3.4. One considers $\mathcal{A}$ and $\mathcal{A}_{1}$ two Banach real algebras such that $\mathcal{A}=\mathcal{A}_{1}{ }^{*}$. One supposes that $F:\left[x_{0}, x_{m-1}\right] \rightarrow \mathcal{A}$ has Fréchet derivatives of all orders on $\left[x_{0}, x_{m-1}\right]$. If $M_{s}=$ $\sup _{x \in\left[x_{0}, x_{m-1}\right]}\left\|F^{(s)}(x)\right\|$ and the sequence $\left\{z_{s}\right\}$ given by

$$
z_{s}=\frac{\left(x_{m-1}-x_{0}\right)^{s} M_{s}}{s !}, \quad s=0,1, \ldots
$$

converges to zero, then $F$ is the sum of its Newton interpolating series at $x_{0}, x_{1}, \ldots, x_{m-1}$ on $\left[x_{0}, x_{m-1}\right]$.

Proof. From (3.7) it follows that

$$
\left\|F(x)-N_{n}(x)\right\| \leq \frac{M_{n+1}}{(n+1) !}\left|u_{n+1}(x)\right|,
$$

and by (2.2) we obtain that

$$
\max _{x \in\left[x_{0}, x_{m-1}\right]}\left|u_{n+1}(x)\right| \leq\left(x_{m-1}-x_{0}\right)^{n+1} .
$$

Now (3.10) and (3.11) imply

$$
\left\|F(x)-N_{n}(x)\right\| \leq \frac{\left(x_{m-1}-x_{0}\right)^{n+1} M_{n+1}}{(n+1) !} .
$$

Hence, $N_{n}(x)$ converges uniformly to $F$ on $\left[x_{0}, x_{m-1}\right]$.

In the last theorem of this section we study the algebra of functions which are the sums of their Newton interpolating series at $x_{0}, x_{1}, \ldots, x_{m-1}$.

If $\mathcal{A}$ is a Banach real algebra, then we denote by $\mathcal{N}\left(x_{0}, x_{1}, \ldots, x_{m-1}\right)$ the set of all functions $F:\left[x_{0}, x_{m-1}\right] \rightarrow \mathcal{A}$ which are representable into a Newton interpolating series at $x_{0}, x_{1}, \ldots, x_{m-1}$. Thus, by Theorem 3.1, every $F \in \mathcal{N}\left(x_{0}, x_{1}, \ldots, x_{m-1}\right)$ is the sum of its Newton interpolating series at $x_{0}, x_{1}, \ldots, x_{m-1}$. We take

$$
F(x)=\sum_{i=0}^{\infty} A_{i} u_{i}(x), \quad G(x)=\sum_{i=0}^{\infty} B_{i} u_{i}(x)
$$

two elements of $\mathcal{N}\left(x_{0}, x_{1}, \ldots, x_{m-1}\right)$. Our objective is to define the addition and the multiplication of $F$ and $G$. We begin with a lemma. 
Lemma 3.5. If $u_{i}$ are given in (2.1), then, for every $i, j \in\{1,2, \ldots, m\}$, there exist nonnegative real numbers $C_{k}(i, j), k \in\{\max \{i, j\}, \max \{i, j\}+1, \ldots, i+j\}$, uniquely defined, such that

$$
u_{i} u_{j}=\sum_{k=\max \{i, j\}}^{i+j} C_{k}(i, j) u_{k} .
$$

Proof. If $j \leq i<m$, then it follows that

$$
u_{i} u_{j}=u_{i} u_{j-1}\left(x-x_{j-1}\right)=u_{i+1} u_{j-1}+\left(x_{i}-x_{j-1}\right) u_{i} u_{j-1}
$$

and if $i=m$, then $u_{i} u_{j}=u_{i+j}$. Now, for every $i$, the induction on $j$ completes the proof of the lemma.

We define addition and multiplication of $F$ and $G$ as follows:

$$
\begin{aligned}
(F+G)(x) & =\sum_{i=0}^{\infty}\left(A_{i}+B_{i}\right) u_{i}(x), \\
(F G)(x) & =\sum_{i=0}^{\infty} P_{i} u_{i}(x),
\end{aligned}
$$

where

$$
P_{i}=\sum_{(\alpha, \beta) \in D(i)} C_{i-(q(\alpha)+q(\beta)) m}(r(\alpha), r(\beta)) a_{\alpha} b_{\beta}
$$

$D(i)=\left\{(\alpha, \beta) \in \mathbb{N}^{2}: \max \{\alpha+q(\beta) m, \beta+q(\alpha) m\} \leq i \leq \alpha+\beta\right\}$ and $C_{k}(s, t)$ are given in (3.14). Let $\bar{x} \in\left[x_{0}, x_{m-1}\right]$ be such that

$$
\left|u_{m}(\bar{x})\right|=\sup _{x \in\left[x_{0}, x_{m-1}\right]}\left|u_{m}(x)\right|
$$

Since $u_{i}(x)>0$, for every $x>x_{m-1}$, and the roots of $u_{i}^{\prime}(x)$ belong to $\left[x_{0}, x_{m-1}\right]$, there exists $z>x_{m-1}$, uniquely determined such that

$$
u_{m}(z)=\left|u_{m}(\bar{x})\right|
$$

We put

$$
\begin{aligned}
& K_{1}=\min _{0 \leq i \leq m-1}\left|u_{i}(\bar{x})\right|, \\
& K_{2}=\max _{0 \leq i \leq m-1}\left|u_{i}(z)\right| .
\end{aligned}
$$


Then, by (2.2),

$$
u_{i}(z)=u_{m}^{q(i)}(z) u_{r(i)}(z)=\left|u_{m}^{q(i)}(\bar{x})\right|\left|u_{r(i)}(\bar{x})\right|\left|\frac{u_{r(i)}(z)}{u_{r(i)}(\bar{x})}\right| \leq \frac{K_{2}}{K_{1}}\left|u_{i}(\bar{x})\right|
$$

If $F(x)=\sum_{i=0}^{\infty} A_{i} u_{i}(x) \in \mathcal{N}\left(x_{0}, \ldots, x_{m-1}\right)$, then the series $\sum_{i=0}^{\infty} A_{i} u_{i}(\bar{x})$ converges absolutely and, by (3.21), the series $\sum_{i=0}^{\infty}\left\|A_{i}\right\| u_{i}(z)$ converges. We define

$$
\|F\|_{\mathcal{N}}=\sum_{i=0}^{\infty}\left\|A_{i}\right\| u_{i}(z)
$$

Theorem 3.6. If the addition and the multiplication are defined by (3.16), then $N\left(x_{0}, \ldots, x_{m-1}\right)$ becomes a real algebra. Furthermore, \|\|$_{\mathcal{N}}$ defined by (3.22) is a norm, and $\mathcal{N}\left(x_{0}, \ldots, x_{m-1}\right)$ becomes a real Banach algebra with respect to \|\|$_{\mathcal{N}}$.

Proof. If $F, G \in \mathcal{N}\left(x_{0}, \ldots, x_{m-1}\right)$, then their Newton interpolating series at $x_{0}, x_{1}, \ldots, x_{m-1}$ converge absolutely on $\left[x_{0}, x_{m-1}\right]$ and converge uniformly to $F$ and $G$, respectively. By (3.16) it follows easily that the Newton interpolating series at $x_{0}, x_{1}, \ldots, x_{m-1}$ of $F+G$ and $F G$ have the same properties. Thus, $\mathcal{N}\left(x_{0}, \ldots, x_{m-1}\right)$ is a real algebra.Since

$$
\left\|u_{i} u_{j}\right\|_{\mathcal{N}^{\prime}}=\left\|\sum_{k=i}^{i+j} C_{k}(i, j) u_{k}\right\|_{\mathcal{N}}=\sum_{k=i}^{i+j} C_{k}(i, j) u_{k}(z)=\left\|u_{i}\right\|_{\mathcal{N}}\left\|u_{j}\right\|_{\mathcal{N}^{\prime}}
$$

for every $i, j \in\{1,2, \ldots, m\}, i \geq j$, it follows that $\|F G\|_{\mathcal{N}} \leq\|F\|_{\mathcal{N}}\|G\|_{\mathcal{N}}$ and $\mathcal{N}\left(x_{0}, \ldots, x_{m-1}\right)$ is a normed algebra.

Now, we will prove that $\mathcal{N}\left(x_{0}, \ldots, x_{m-1}\right)$ is a real Banach algebra. Consider $\left\{F_{n}=\right.$ $\left.\sum_{j=0}^{\infty} A_{j, n} u_{j}\right\}_{n \in \mathbb{N}}$ a Cauchy sequence of $\mathcal{N}\left(x_{0}, \ldots, x_{m-1}\right)$. Let $\epsilon>0$ be arbitrary. Then there exists $N(\epsilon) \in \mathbb{N}$ such that, for all $n \geq N(\epsilon)$ and $p=1,2, \ldots,\left\|F_{n+p}-F_{n}\right\|_{\mathcal{N}}<\epsilon$. From (3.22), we obtain

$$
\sum_{j=0}^{\infty}\left\|A_{j, n+p}-A_{j, n}\right\| u_{j}(z)<\epsilon
$$

Thus, for each fixed $j, n \geq N(\epsilon)$ and $p=1,2, \ldots,\left\|A_{j, n+p}-A_{j, n}\right\| u_{j}(z)<\epsilon$. Hence, $\left\{A_{j, n}\right\}_{n \in \mathbb{N}}$ is a Cauchy sequence in $\mathcal{A}$ and, since $\mathcal{A}$ is a complete algebra, this sequence converges. We set $A_{j}=\lim _{n \rightarrow \infty} A_{j, n}$, and we consider the formal series

$$
F=\sum_{j=0}^{\infty} A_{j} u_{j}
$$

We have to show that $F \in \mathcal{N}\left(x_{0}, x_{1}, \ldots, x_{m-1}\right)$ and $\lim _{n \rightarrow \infty} F_{n}=F$. From (3.24),

$$
\sum_{j=0}^{s}\left\|A_{j, n+p}-A_{j, n}\right\| u_{j}(z)<\epsilon
$$


Fix now some $n \geq N(\epsilon)$ and let $p \rightarrow \infty$ and then $s \rightarrow \infty$. Hence, for all $n \geq N(\epsilon)$, we obtain

$$
\sum_{j=0}^{\infty}\left\|A_{j}-A_{j, n}\right\| u_{j}(z) \leq \epsilon
$$

Since, for $x \in\left[x_{0}, x_{m-1}\right], n_{0} \geq N(\epsilon), p^{\prime}=0,1, \ldots,\left\|\sum_{j=i}^{i+p^{\prime}} A_{j} u_{j}(x)\right\| \leq \sum_{j=i}^{\infty}\left\|A_{j}-A_{j, n_{0}}\right\| u_{j}(z)+$ $\sum_{j=i}^{\infty}\left\|A_{j, n_{0}}\right\| u_{j}(z)$ and $F_{n_{0}} \in \mathcal{N}\left(x_{0}, \ldots, x_{m-1}\right)$, from (3.27), it follows that $F$ is a function defined on $\left[x_{0}, x_{m-1}\right]$ and it is representable into the Newton interpolation series (3.25). Thus, $F \in$ $\mathcal{N}\left(x_{0}, \ldots, x_{m-1}\right)$. Finally, from (3.27), $\left\|F-F_{n}\right\|_{\mathcal{N}} \leq \epsilon$ and $\lim _{n \rightarrow \infty} F_{n}=F$.

Remark 3.7. Let $\mathcal{A}[x]$ be the ring of polynomials with coefficients in $\mathcal{A}$, where $x$ commutes with the elements of $\mathcal{A}$. With respect to the topology defined by the norm given in (3.22), $\mathscr{A}[x]$ is a dense subset of $\mathcal{N}\left(x_{0}, \ldots, x_{m-1}\right)$. If $F$ satisfies the hypotheses of Theorem 3.4 and its Newton interpolating series converges absolutely at $\bar{x}$ defined in (3.18), then $F \in$ $\mathcal{N}\left(x_{0}, \ldots, x_{m-1}\right)$. Hence, it follows that if $F: I \rightarrow \mathbb{R}$, where $I$ is an open interval which contains $x_{i}$, for every $i$, and its Taylor series at $x_{0}$ converges on $I$, then the restriction of $F$ to $\left[x_{0}, x_{m-1}\right]$ belongs to $\mathcal{N}\left(x_{0}, \ldots, x_{m-1}\right)$. However, $\mathcal{N}\left(x_{0}, \ldots, x_{m-1}\right)$ contains also functions which are not Fréchet differentiable on $\left[x_{0}, x_{m-1}\right]$. For example, we take $\mathcal{A}=\mathbb{R}, m=2, x_{0}=-1$, $x_{1}=1$ and $F(x)=|x|$. Then,

$$
F(x)=\sqrt{1-\left(1-x^{2}\right)}=1+\sum_{k=1}^{\infty} \frac{(-1)^{k+1} \prod_{i=1}^{k-2}(k+i)}{(k-1) ! 2^{2 k-1}}\left(x^{2}-1\right)^{k}
$$

for $x \in[-1,1]$, which implies that $F \in \mathcal{N}(-1,1)$.

\section{References}

[1] A. B. Shidlovsky, Transcendental Numbers, Nauka, Moscow, Russia, 1987.

[2] G. Groza and N. Pop, "Approximate solution of multipoint boundary value problems for linear differential equations by polynomial functions," Journal of Difference Equations and Applications, vol. 14, no. 12, pp. 1289-1309, 2008.

[3] F. A. Costabile and E. Longo, "A Birkhoff interpolation problem and application," Calcolo, vol. 47, no. 1, pp. 49-63, 2010.

[4] G. Groza, N. Popescu, and A. Zaharescu, "Norms on $R\left[X_{1}, \ldots, X_{r}\right]$ Which Are Multiplicative on R," Results in Mathematics, vol. 51, no. 3-4, pp. 229-247, 2008.

[5] R. A. DeVore and G. G. Lorentz, Constructive Approximation, vol. 303 of Fundamental Principles of Mathematical Sciences, Springer, Berlin, Germany, 1993. 


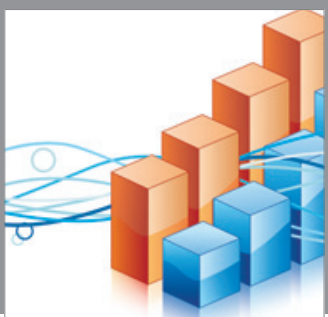

Advances in

Operations Research

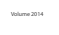

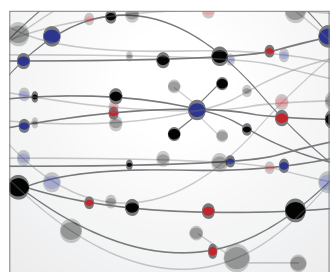

\section{The Scientific} World Journal
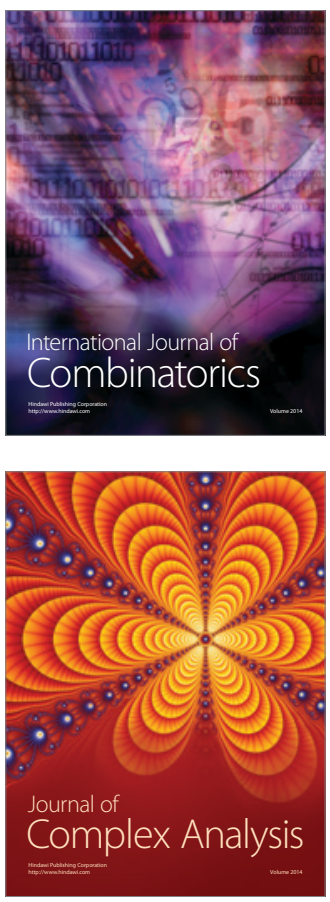

International Journal of

Mathematics and

Mathematical

Sciences
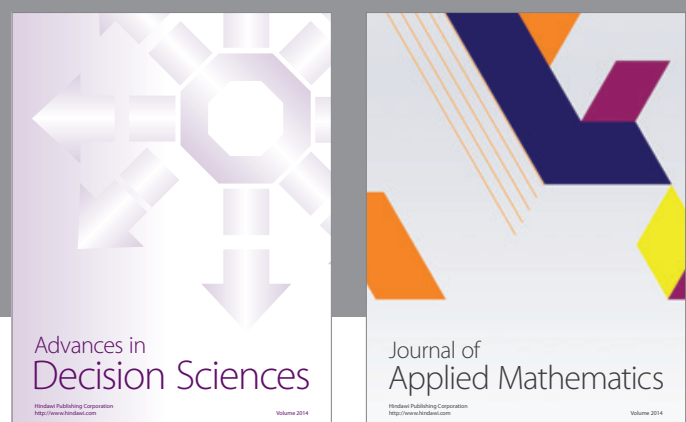

Journal of

Applied Mathematics
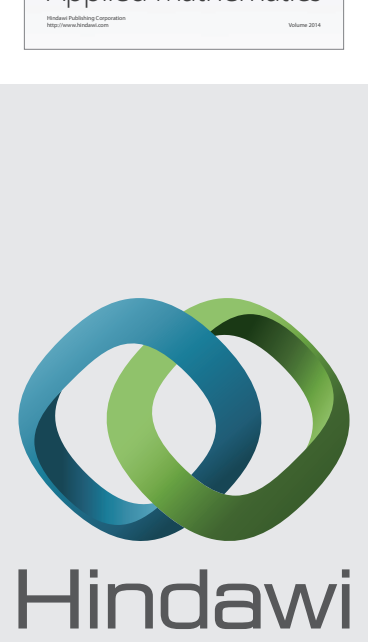

Submit your manuscripts at http://www.hindawi.com
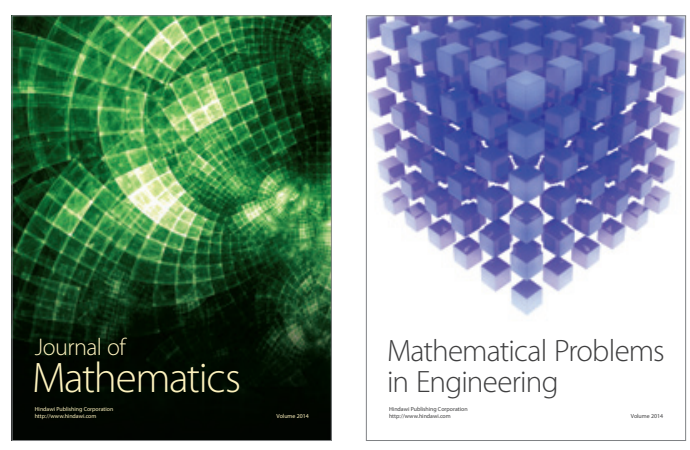

Mathematical Problems in Engineering
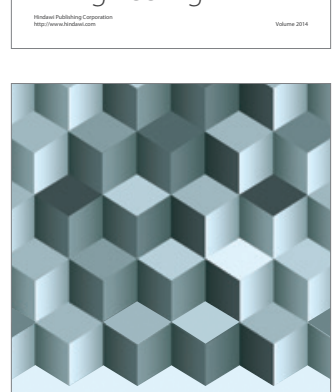

Journal of

Function Spaces
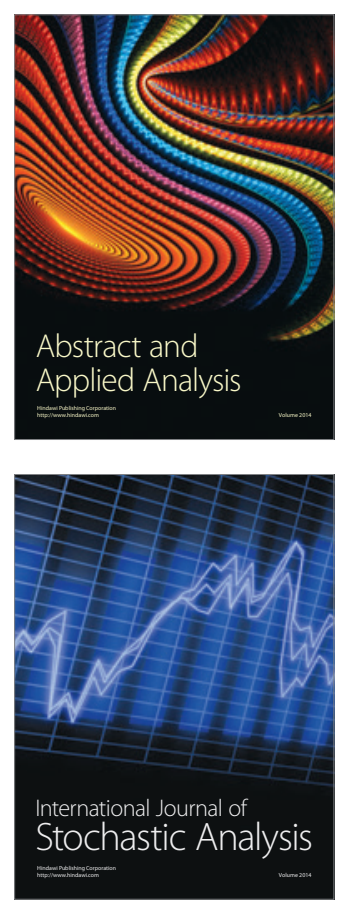

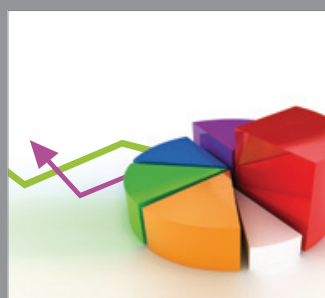

ournal of

Probability and Statistics

Promensencen
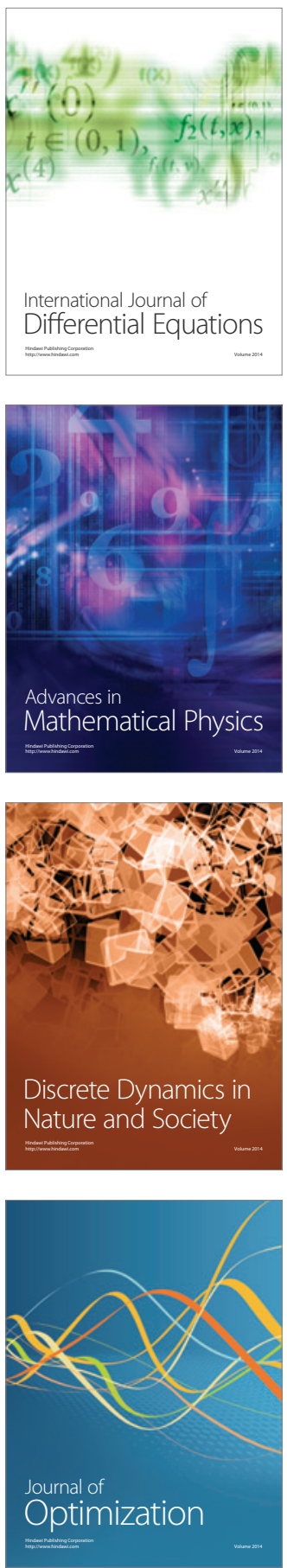